\title{
A social work study for comparison of thought action-fusion and anxiety sensitivity among normal student versus obsessive compulsive symptom in high school Esfahan city
}

\author{
Reyhaneh Shojaei Jeshvaghani $^{a^{*}}$, Farzaneh Niknejadi ${ }^{b}$ and Yousef Gorji ${ }^{b}$
}

${ }^{a}$ MS Student, Counseling Department, Islamic Azad University of Khomeinishahr, Khomeinishahr Branch, Daneshjou Blvd, Iran

${ }^{b}$ Assistant Professor, Counseling Department, Islamic Azad University of Khomeinishahr,Khomeinishahr Branch, Daneshjou Blvd, Iran

\begin{tabular}{l}
\hline A R T I C L E I N F O \\
\hline Article history: \\
Received August 25, 2012 \\
Received in revised format \\
25 September 2012 \\
Accepted 4 October 2012 \\
Available online \\
October 5 2012 \\
\hline Keywords: \\
Obsessive-Compulsive Disorder \\
Thought-action fusion \\
OCD \\
TAF
\end{tabular}

\section{A B S T R A C T}

The purpose of this study is to compare thought - action fusion and anxiety sensitivity among girls and boys adolescents having obsessive-compulsive symptoms in clinical and nonclinical extent. The survey selects 385 high school students in city of Esfahan, Iran and distributes a questionnaire among them. All questions are designed in Likert scale and participants are divided into two groups of with and without OCD. The results show that there is a significant and positive difference in thought - action fusion and anxiety sensitivity among girls and boys adolescents having or symptoms in clinical and nonclinical l extent $(\mathrm{P}<0.01)$.

(c) 2012 Growing Science Ltd. All rights reserved.

\section{Introduction}

During the past few years, there has been growing interest on learning more about obsessivecompulsive disorder (OCD) and thought-action fusion (TAF). An obvious consequence of OCD appears as anxiety and depression. Steketee et al. (1987) explained that OCD is more functionally debilitating than other anxiety disorders, but greater psychopathy in the family background was not supported. Amir et al. (2001) modified the TAF to include a scale, which evaluated the "likelihood of events happening to others" as well as ratings of the responsibility and cost for having these thoughts. They found that individuals with OC symptoms gave higher ratings to the likelihood of negative events happening because of their negative thoughts. Individuals with OC symptoms also ranked the likelihood that they could protect from harm by their positive thoughts higher than did individuals

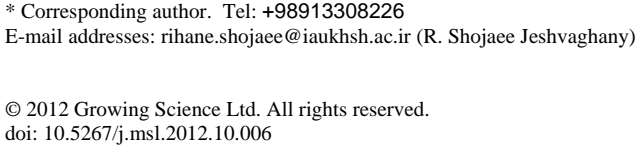


without OC symptoms. Their results recommended that the role of TAF in OCs may extend to exaggerated beliefs about thoughts based on the reduction of harm.

Muris et al. (2001) investigated TAF in a large sample of normal adolescents. They asked the sample participants to complete the TAF Questionnaire for Adolescents (TAFQ-A) and scaled measuring trait anxiety, symptoms of obsessive-compulsive disorder, other anxiety disorders, and depression. Their results indicated that the TAFQ-A was a reliable instrument for evaluating two dimensions of TAF, viz. According to their results, symptoms of OCD and generalized anxiety remained substantially associated with TAF. McWilliams et al. (2000) that the AS Social Concerns factor was distinct from negative evaluation sensitivity (NES) and the other lower-order components of AS. However, other analyses and higher-order principle component analysis revealed that the AS Social Concerns factor taps a blend of AS and NES as well as something unique and distinct from both global AS and NES.

Rassin et al. (2000) explained that there are strong similarities in content between the obsessions and compulsions, which could characterize OCD and nonclinical obsessions and compulsions. However, clinical and nonclinical obsessions and compulsions were found to be different with respect to characteristics like frequency, discomfort, intensity and elicited resistance. They invoked two different concepts to describe how normal obsessions and compulsions could develop into clinical phenomena. The results described that TAF contributed to OCD. The study explored how TAF and thought suppression could interact in the development of obsessive-compulsive symptoms. Their results recommended that TAF triggers thought suppression, while thought suppression, in turn, promotes obsessive-compulsive symptoms.

According to Maller and Reiss (1992), there are some evidences for the stability of anxiety sensitivity over a long period of time and for the exceptionally strong relationships over time among panic attacks, anxiety sensitivity and panic disorder. Kamieniecki et al. (1997) studied the cognitive model of panic disorder by studying two issues. The first was whether panic patients misinterpret bodily sensations and the second was whether panic patients were able to incorporate benign subsequent explanations for bodily sensations. Their results provided support for the cognitive theory of panic disorder.

Essau et al. (2010), for instance, investigated the factor structure, reliability, and validity of the German translation of the Childhood Anxiety Sensitivity Index (CASI). They did a survey among a total of 1292 adolescents and analyzed the results using the Schmid-Leiman. They reported that the CASI to have one higher order factor and three orthogonal lower order factors. CASI scores correlated substantially with anxiety symptoms and with overall difficulties. They also used regression techniques and the results indicated that CASI influenced on contribution in predicting anxiety even after controlling for variance due to general difficulties.

Schmidt et al. (2010) explained that evidence recommends that anxiety sensitivity (AS) predicts subsequent development of anxiety symptoms and panic attacks and clinical syndromes in adult samples. They implemented a study to detect whether AS similarly could act as a vulnerability factor in the pathogenesis of anxiety symptoms among youth in early adolescence (ages 9-13). They reported some results, which were consistent with the adult literature and expectancy theory and concluded that AS appeared to act as a risk factor for anxiety symptoms in youth.

Stewart et al. (1997) performed an investigation on gender differences in dimensions of anxiety sensitivity. They provided some support for the empirical distinction of the three lower-order dimensions of AS, and some additional evidence for the theoretical hierarchical structure of the Anxiety Sensitivity Index. Their results also recommended that males and females differ on these various AS dimensions in different ways consistent with sex impact socialization practices. 


\section{The proposed study}

The research purpose is to study and compare thought - action fusion and anxiety sensitivity among girls and boys adolescents having obsessive-compulsive symptoms in clinical and nonclinical extent. The following formula to calculate the minimum number of sample size,

$n=\frac{N \times z_{\alpha / 2}^{2} \times p \times q}{\varepsilon^{2} \times(N-1)+z_{\alpha / 2}^{2} \times p \times q}$,

where $N$ is the population size, $p=1-q$ represents the yes/no categories, $z_{\alpha / 2}$ is CDF of normal distribution and finally $\varepsilon$ is the error term. Since we have $p=0.5, z_{\alpha / 2}=1.96$ and $N=16588$, the number of sample size is calculated as $n=385$. The survey selects 385 high school students in city of Esfahan, Iran and distributes a questionnaire among them. All questions of the questionnaire were in Likert scale from very low to very high. Fig. 1 shows details of the number of participants in our survey.

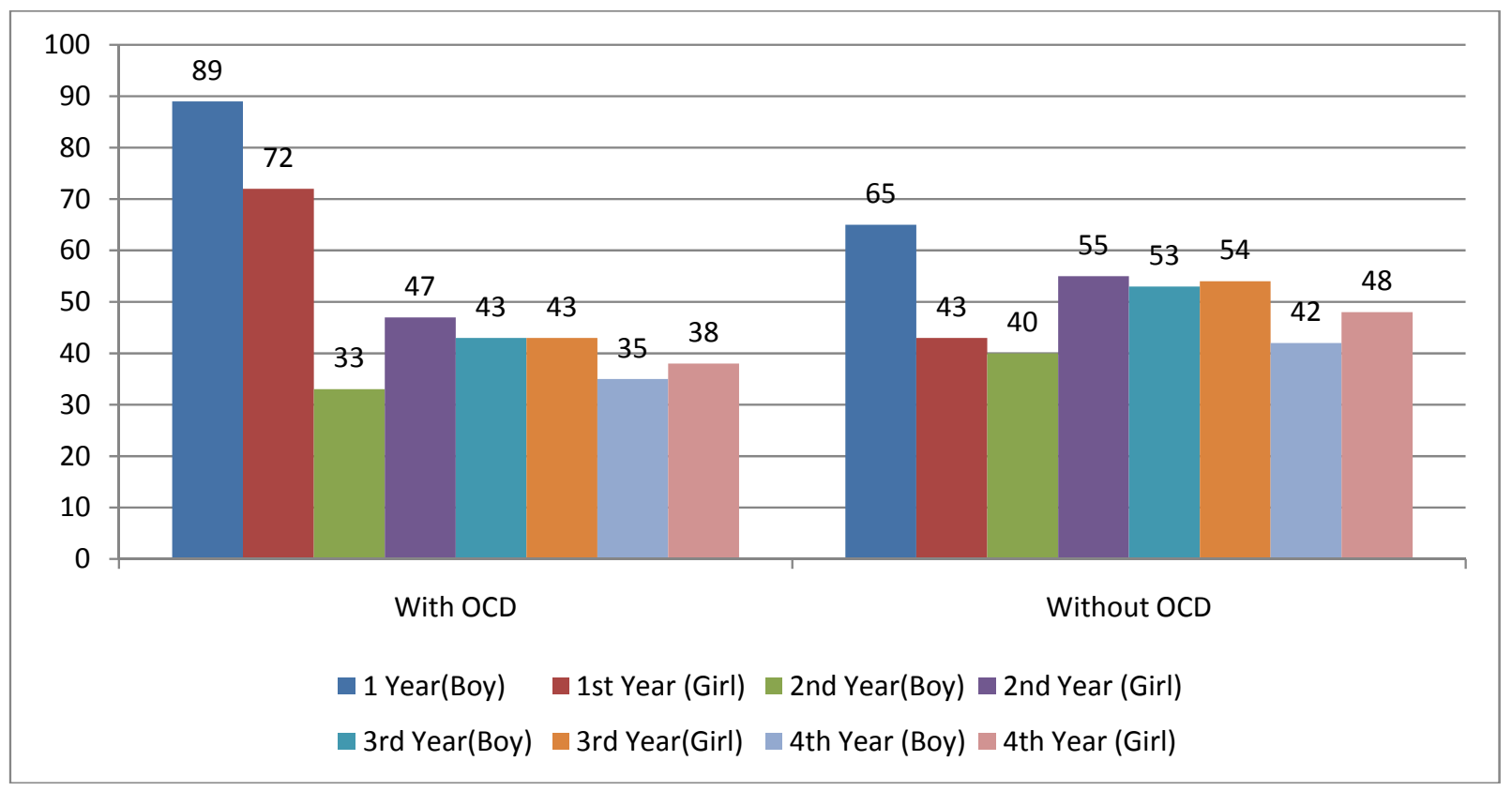

Fig. 1. Frequencies of teenage girls and boys with and without OCD

As we can observe from the results of Fig. 1, first year high school teenage boys are more than teenage girls in both groups but among the second to the fourth years high school teenagers there is not much differences.

The proposed study of this paper considers the following hypotheses,

1. There is a positive relationship among teenagers between obsessive-compulsive disorder (OCD) and thought- action fusion (TAF) in clinical and nonclinical extent.

2. There is a meaningful relationship for anxiety sensitivity (AC), among teenagers with OCD, in clinical and nonclinical extent. 
3. There is a positive relationship among teenage boys between obsessive-compulsive disorder (OCD) and thought- action fusion (TAF) in clinical and nonclinical extent.

4. There is a positive relationship among teenage girls between obsessive-compulsive disorder (OCD) and thought- action fusion (TAF) in clinical and nonclinical extent.

5. There is a meaningful relationship for anxiety sensitivity (AC), among teenage boys with OCD, in clinical and nonclinical extent.

6. There is a meaningful relationship for anxiety sensitivity (AC), among teenage girls with OCD, in clinical and nonclinical extent.

\section{The results}

In this section, we present details of our findings associated with six hypotheses.

\subsection{The relationship between OCD and TAF among teenagers}

Table 1 shows details of our findings on the relationship between OCD and TAF among all teenagers who participated in our survey.

\section{Table 1}

The results of our testing for the relationship between OCD and TAF

\begin{tabular}{lccc}
\hline Group & Mean rank & Z & P-Value \\
\hline With OCD & 479.97 & 9.72 & 0.0001 \\
Without OCD & 321.03 & & \\
\hline
\end{tabular}

As we can observe form the results of Table 1, there is a meaningful TAF difference between teenagers with and without OCD when the level of significance is one percent.

\subsection{The relationship between AC and TAF among teenagers}

Table 2 shows details of our findings on the relationship between AC and TAF among all teenagers who participated in our survey.

Table 2

The results of our testing for the relationship between AC and TAF

\begin{tabular}{lccc}
\hline Group & Mean rank & Z & P-Value \\
\hline With OCD & 513.74 & 14.12 & 0.001 \\
Without OCD & 283.40 & & \\
\hline
\end{tabular}

As we can observe form the results of Table 2, there is a meaningful AC and TAF difference between teenagers with and without OCD when the level of significance is one percent.

\subsection{The relationship between OCD and TAF among teenage boys}

Table 3 shows details of our findings on the relationship between OCD and TAF among all teenage boys who participated in our survey.

\section{Table 3}

The results of our testing for the relationship between OCD and TAF among teenage boys

\begin{tabular}{lccc}
\hline Group & Mean rank & Z & P-Value \\
\hline With OCD & 228.22 & 4.79 & 0.001 \\
Without OCD & 172.178 & & \\
\hline
\end{tabular}


As we can observe form the results of Table 3, there is a meaningful TAF difference between teenage boys with and without OCD when the level of significance is one percent.

\subsection{The relationship between OCD and TAF among teenage girls}

Table 4 shows details of our findings on the relationship between OCD and TAF among all teenage girls who participated in our survey.

\section{Table 4}

The results of our testing for the relationship between OCD and TAF among teenage girls

\begin{tabular}{lccc}
\hline Group & Mean rank & $Z$ & P-Value \\
\hline With OCD & 281.85 & 8.88 & 0.001 \\
Without OCD & 149.15 & & \\
\hline
\end{tabular}

As we can observe form the results of Table 4, there is a meaningful TAF difference between teenage girls with and without OCD when the level of significance is one percent.

\subsection{The relationship between AC and TAF among teenage boys}

Table 5 shows details of our findings on the relationship between AC and TAF among all teenagers who participated in our survey.

\section{Table 5}

The results of our testing for the relationship between AC and TAF among teenage boys

\begin{tabular}{lccc}
\hline Group & Mean rank & Z & P-Value \\
\hline With OCD & 257.58 & 10.25 & 0.001 \\
Without OCD & 139.53 & & \\
\hline
\end{tabular}

As we can observe form the results of Table 5, there is a meaningful TAF difference between teenage boys with and without OCD when the level of significance is one percent.

\subsection{The relationship between AC and TAF among teenage girls}

Table 5 shows details of our findings on the relationship between AC and TAF among all teenage girls who participated in our survey.

\section{Table 6}

The results of our testing for the relationship between AC and TAF among teenage girls

\begin{tabular}{lccc}
\hline Group & Mean rank & Z & P-Value \\
\hline With OCD & 257.53 & 9.86 & 0.001 \\
\hline Without OCD & 143.47 & & \\
\hline
\end{tabular}

As we can observe form the results of Table 6, there is a meaningful TAF difference between teenage girls with and without OCD when the level of significance is one percent.

\section{Conclusion}

In this paper, we have presented and empirical study to compare thought - action fusion and anxiety sensitivity among girls and boys adolescents having obsessive-compulsive symptoms in clinical and nonclinical extent. We have designed and distributed a questionnaire among 384 teenage students 
who attended high schools in city of Esfahan, Iran. The results of our survey confirmed that there was a meaningful difference between teenagers with and without OCD when the level of significance is one percent and the results were also valid in separate genders of female and male. There was also a meaningful difference between teenagers' OCD and TAF characteristics among who participated in our survey. Again, the results hold when the survey was performed between teenage girls and teenage boys, separately.

\section{Acknowledgment}

Islamic Azad University of Khomeinishahr financially supported this research and authors would like to acknowledge the support.

\section{References}

Amir, N., Freshman, M., Ramsey, B., Neary, E., \& Brigidi, B. (2001). Thought-action fusion in individuals with OCD symptoms. Behaviour Research and Therapy, 39(7), 765-776.

Essau, C.A., Sasagawa, S., \& Ollendick, T.H. (2010). The facets of anxiety sensitivity in adolescents. Journal of Anxiety Disorders, 24(1), 23-29.

Kamieniecki, G.W., Wade, T., \& Tsourtos, G. (1997). Interpretive bias for benign sensations in panic disorder with agoraphobia. Journal of Anxiety Disorders, 11(2), 141-156.

McWilliams, L.A., Stewart, S.H., \& MacPherson, P.S.R. (2000). Does the social concerns component of the Anxiety Sensitivity Index belong to the domain of anxiety sensitivity or the domain of negative evaluation sensitivity? Behaviour Research and Therapy, 38(10), 985-992.

Maller, R. G., \& Reiss, S. (1992). Anxiety sensitivity in 1984 and panic attacks in 1987. Journal of Anxiety Disorders, 6(3), 241-247.

Muris, P., Meesters, C., Rassin, E., Merckelbach, H., \& Campbell, J. (2001). Thought-action fusion and anxiety disorders symptoms in normal adolescents. Behaviour Research and Therapy, 39(7), 843-852.

Rassin, E., Muris, P., Schmidt, H., \& Merckelbach, H. (2000). Relationships between thought-action fusion, thought suppression and obsessive-compulsive symptoms: a structural equation modeling approach. Behaviour Research and Therapy, 38(1), 889-897

Schmidt, N.B., Keough, M.E., Mitchell, M.A., Reynolds, E.K., MacPherson, L., Zvolensky, M.J., \& Lejuez, C.W. (2010). Anxiety sensitivity: Prospective prediction of anxiety among early adolescents. Journal of Anxiety Disorders, 25(5), 503-508.

Steketee, G., Grayson, J.B., \& Foa, E.B. (1987). A comparison of characteristics of obsessivecompulsive disorder and other anxiety disorders. Journal of Anxiety Disorders, 1(4), 325-335.

Stewart, S.H., Taylor, S., \& Baker, J.M. (1997). Gender differences in dimensions of anxiety sensitivity. Journal of Anxiety Disorders, 11(2), 179-200 